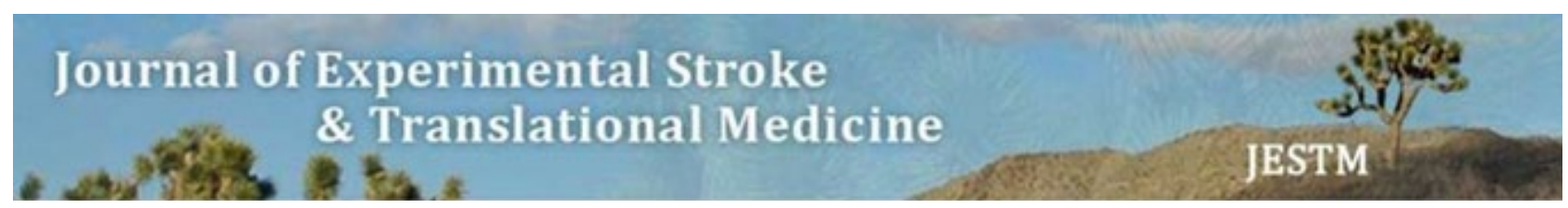

\title{
Comparison of Pain and Functional State and Quality of Life of Total Knee Replacement Patient
}

\author{
July 27, $2016 \cdot$ Volume $9 \cdot$ Original Research \\ Ji-yeon Lee ${ }^{1}$, Jung-Hee Kim², Eun-Jung Jung ${ }^{2}$ and Byounghee Lee ${ }^{3 *}$ \\ ${ }^{1}$ Graduate School of Physical Therapy, Sahmyook University, Seoul, Republic of Korea \\ 2Department of Physical Therapy, Andong Science College, Seoul, Republic of Korea \\ ${ }^{3}$ Department of Physical Therapy, Sahmyook University, Seoul, Republic of Korea
}

Article Citation: Lee, Kim, Jung, Lee Comparison of Pain and Functional State and Quality of Life of Total Knee Replacement Patient. J Exp Stroke Transl Med. 2016 December. Online access at www.jestm.com

Correspondence should be sent to: Byoung-Hee Lee, Department of Physical Therapy, Sahmyook University, Hwarangro-815, Nowongu, Seoul, Republic of Korea, Tel: 8223399634, Fax: 8223991639, E-Mail: 3679@syu.ac.kr

\section{Abstract}

Purpose: To provide a basic data for understanding and guideline for management of total knee replacement patients through the comparisons of functional level and quality of life between total knee replacement patient and non-total knee replacement patient.

Methods: For a total of 135 knee arthritis patients evaluated for functional levels and quality of life through the range of motion, angle of genuvalgus, VAS, KSS, KSFS, HSS and WOMAC.

Results: TKR group showed a more significant differences in pain, ROM, angle of contracture, functional level and quality of life than non-TKR group $(p<0.05)$. The Both side arthritis group showed a more significant differences in ROM, functional level and quality of life than uni-side arthritis group $(p<0.05)$.

Conclusion: To increasing of functional level and quality of life after TKR, self-care program including muscle strengthening has to conduct.

Key Words: Total knee replacement; knee arthritis; quality of life.

\section{Introduction}

Knee osteoarthritis is a disease that causes pain, functional limitation and disability and its incidence rate is gradually rising with an increase in the elderly population (Felson \& Anderson, 1987; McKay, Prapavessis \& Doherty, 2012). A total knee replacement is an effective surgical intervention for the patients with knee osteoarthritis by reducing pain in the knee joints and enhancing the quality of life and joint functions for a smoother gait. A study of the cohort of patients with total knee replacement showed a significant increase in the Short Form-36 Health Survey (Gidwani, Tauro, Whitehouse \& Newman, 2003; Keurentjes et al., 2013). However, all the prognoses of the patients with total knee are positive. Some patients with total knee replacement show little improvement in the knee functions after the operation (Dickstein, Heffes, Shabtai \& Markowitz, 1998) and complain of a decrease in the knee extension torque, range of motion and functional mobility, and a difficulty in carrying out daily functional tasks (Holm, et al., 2010; McClelland, Webster, Feller, \& Menz, 2010). Such functional limitations are mainly related with weaknesses of muscle strength of lower extremities so even after the operation, the weakness in the knee extensor and flexor may continue into a few months or even years (Valtonen, Poyhonen, Sipila, \& Heinonen, 2010). There are many studies being conducted on the knee function of patients with total knee replacement at various levels, nonetheless, there are too few studies to compare and analyze the knee function of the surgical site, based on whether or not research subjects had total knee replacement (TKR) and whether they had TKR on one or both knees. Therefore, the present study aims to explore the differences in knee function between the subjects who had TKR and those who did not, and between the subjects who had TKR on one knee and those who had on both knees in order to gather underlying information needed to understand the clinical features of patients with TKR.

\section{Methods}

\section{Subjects}

Participants for this study were selected from among 135 patients with knee osteoarthritis who were hospitalized at the $\mathrm{H}$ Hospital in South Korea. The inclusion criteria were as follows: patients who have no other operation except knee surgery, no functional limitation with nervous system disorder or other disease and the ability to understand the purpose of the study and will fully participate in it. The exclusion criteria were as follows: patients who have other disease except knee arthritis, patient who reoperation within one year.

The VAS used for evaluation of knee pain of subject. During the study, the patients rated their pain on the 100 mm horizontal linear scale of 0 to 10 with a higher score indicating more severe pain (Lundblad et al., 2012). During knee flexion and extension, the knee's range of motion was measured using goniometer when pain was felt. And the tibiofemoral angle was measured on anterior-posterior $\mathrm{x}$-ray images in a standing position. The function of knee joint was evaluated based on functional activities using the Knee Society Knee Score (KSFS) which encompasses pain, range of motion, stability, flexion contracture and distance of walking, and the Knee Society Function Score (KSFS) which includes stair climbing (Lingard, Katz, Wright, Wright \& Sledge, 2001). 
The Hospital Severity Score (HSS) was used to evaluate the clinical state of patients with total knee replacement (Ikejiani, Leighton \& Petrie, 2000; Russo et al., 2008). Moreover, the Short Form 36 (SF-36) health survey was carried out to assess the quality of life and consists of a total of eight subcategories of physical function(PF), role physics(RP), bodily pain(BP), general health(GH), vitality(VT), social function(SF), restriction emotion (RE) and mental health (MH). The score of each subcategory was converted to a scale of $0-100$ with a higher score indicating better health (Ko et al., 2011; McKay et al., 2012). The Western Ontario and McMaster Universities Arthritis Index (WOMAC) was used along with SF-36 as tools to evaluate knee functions and the patients' quality of life (Nunez et al., 2011). It comprises a total of 24 subcategories under the key categories of pain, stiffness and physical functions. Scores are on the scale of 0 to 4 with four being the highest score and low scores indicate low levels of pain and knee functions. WOMAC reliability is in the range of 0.77-0.83 during measurement and re-measurement (McKay et al., 2012).

\section{Statistical analysis}

All statistical analyses in this study were performed using the Statistical Package for the Social Sciences (SPSS) version 19.0. The general characteristics of participants are expressed as mean and standard deviation, using the descriptive analysis function in SPSS. To compare of differences between the before and after of operation, independent $t$ test was performed. The level of statistical significance was set at $\mathrm{p} \leq 0.05$ for all tests.

\section{Results}

As there were few differences between the groups, experiments were conducted in homogeneous condition (Table 1). The ROM of knee flexion of left leg in the TKR group the non-TKR group were 127.94 degrees and 134.54 degrees, respectively, with significant differences between the groups $(p<0.05)$ (Table 2). The VAS scores of subjects in the TKR group and the non-TKR group were 7.00 and 5.85 years, respectively with significant differences between the groups $(p<0.05)$. The KSS scores of both legs in the TKR group and the non-TKR group were 38.85 (left leg of TKR) and 38.86 (right side of TKR), 46.60 (left side of non-TKR) and 48.27 (right of non-TKR), respectively with significant differences between the groups $(p<0.05)$.

\begin{tabular}{|l|l|l|l|}
\hline & TKR $(\boldsymbol{n}=\mathbf{7 0})$ & Non-TKR $(\boldsymbol{n}=\mathbf{6 5})$ & $\mathbf{t}(\boldsymbol{p})$ \\
\hline Age $($ year $)$ & $71.04 \pm 7.31^{\mathrm{a}}$ & $55.51 \pm 5.06$ & $14.448(0.000)$ \\
\hline Height $(\mathbf{c m})$ & $151.84 \pm 5.94$ & $157.62 \pm 6.47$ & $-5.403(0.000)$ \\
\hline Weight $(\mathbf{k g})$ & $62.79 \pm 8.95$ & $67.59 \pm 9.08$ & $-3.090(0.002)$ \\
\hline BMI $\left(\mathbf{k g} / \mathbf{m}^{2}\right)$ & $27.20 \pm 3.33$ & $27.18 \pm 3.31$ & $0.030(0.976)$ \\
\hline aMean \pm SD, TKR: Total Knee Replacement, BMI: Body Mass Index & \\
\hline
\end{tabular}

Table 1.

General characteristics of participants by TKR and non-TKR (N=135)

\begin{tabular}{|l|l|l|l|l|}
\hline \multirow{4}{*}{ Lt. leg } & Range of extension & TKR $(\boldsymbol{n}=\mathbf{7 0})$ & non TKR $(\boldsymbol{n}=\mathbf{6 5})$ & $\mathbf{t}(\boldsymbol{p})$ \\
\cline { 2 - 5 } & Range of flexion & $4.68 \pm 4.57^{\mathrm{a}}$ & $2.85 \pm 2.50$ & $2.834(0.005)$ \\
\cline { 2 - 5 } & Angle of flexion contracture & $127.94 \pm 7.81$ & $131.54 \pm 5.92$ & $-2.946(0.004)$ \\
\cline { 2 - 5 } & Range of varus & $0.00 \pm 0.00$ & $0.77 \pm 2.69$ & $-2.309(0.024)$ \\
\hline \multirow{2}{*}{ Rt. Leg } & Range of extension & $-18.60 \pm 4.10$ & $-14.51 \pm 7.88$ & $-3.703(0.000)$ \\
\cline { 2 - 5 } & Range of flexion & $4.77 \pm 4.00$ & $3.00 \pm 3.16$ & $1.601(0.114)$ \\
\cline { 2 - 5 } & Angle of flexion contracture & $126.77 \pm 9.82$ & $130.33 \pm 7.43$ & $-1.319(0.191)$ \\
\hline & Range of varus & $0.00 \pm 0.00$ & $0.67 \pm 2.58$ & $-1.000(0.334)$ \\
\hline aMean \pm SD & & $-18.22 \pm 3.74$ & $-11.47 \pm 8.75$ & $-2.926(0.010)$ \\
\hline
\end{tabular}

Table 2.

Differences of range of motion by TKR and non-TKR $(\mathrm{N}=135)$

The KSFS scores of both legs in the TKR group and the non-TKR group were 32.50 (left leg of TKR) and 33.50(right side of TKR), 45.92 (left side of non-TKR) and 46.00 (right of non-TKR), respectively with significant differences between the groups $(p<0.05)$. The HSS scores of both legs in the TKR group and the non-TKR group were 65.27 (left leg of TKR) and 64.91(right side of TKR), 71.05 (left side of nonTKR) and 71.40 (right of non-TKR), respectively with significant differences between the groups $(p<0.05)$. The WOMAC scores of the TKR group and the non-TKR group were 71.20 and 76.03, respectively, with significant differences between the groups $(p<0.05)$.

The SF-36 scores of the TKR group and the non-TKR group were 43.29 and 46.68 , respectively, with significant differences between the groups $(p<0.05)$. The SF-36 PCS scores of the TKR group and the non-TKR group were 30.14 and 32.54 , respectively, with significant differences between the groups $(p<0.05)$. The SF-36 MCS scores of the TKR group and the non-TKR group were 38.76 and 39.22 , respectively, with significant differences between the groups $(p<0.05)($ Table 3$)$.

\begin{tabular}{|l|l|l|l|l|}
\hline & & TKR $(\boldsymbol{n}=\mathbf{7 0})$ & Non-TKR $(\boldsymbol{n = 6 5})$ & $\mathbf{t}(\boldsymbol{p})$ \\
\hline VAS & & $7.00 \pm 1.10$ & $5.85 \pm 1.28$ & $5.627(0.000)$ \\
\hline Left leg & KSS & $38.85 \pm 5.58$ & $46.60 \pm 8.60$ & $-6.048(0.000)$ \\
\cline { 2 - 4 } & KSFS & $32.50 \pm 13.90$ & $45.92 \pm 14.52$ & $-5.317(0.000)$ \\
\hline & HSS & $65.27 \pm 6.66$ & $71.05 \pm 6.45$ & $-4.961(0.000)$ \\
\hline
\end{tabular}




\begin{tabular}{|l|l|l|l|l|}
\hline Right leg & LSS & $38.36 \pm 5.80$ & $48.27 \pm 11.59$ & $-3.217(0.005)$ \\
\cline { 2 - 4 } & KSFS & $33.05 \pm 14.19$ & $46.00 \pm 16.71$ & $-3.076(0.003)$ \\
\hline HSS & $64.91 \pm 6.11$ & $71.40 \pm 2.58$ & $-3.442(0.001)$ \\
\hline SF-36 & & $71.20 \pm 5.94$ & $76.03 \pm 6.71$ & $-4.433(0.000)$ \\
\hline SF-36 PCS & $43.29 \pm 3.52$ & $46.68 \pm 4.67$ & $-4.788(0.000)$ \\
\hline SF-36 MCS & $30.14 \pm 2.27$ & $32.54 \pm 3.11$ & $-5.087(0.000)$ \\
\hline
\end{tabular}

aMean \pm SD, VAS; Visual Analogue Scale, KSS; Knee Society Score, KSFS; Knee Society Function Score, HSS; Hospital for Special Surgery, WOMAC; Western Ontario McMaster Universities Osteoarthritis Index, SF-36; Short Form-35 Health Survey, PCS; Physical Component Summary, MCS; Mental Component Summary

Table 3.

The comparison of pain, function, QOL by TKR and non-TKR ( $N=135)$

The average ages of subjects in the uniside arthritis group and the both side arthritis group were 59.58 years and 66.95 years, respectively. The BMI score of subjects in the uniside arthritis group and the both side arthritis group were $36.96 \mathrm{~kg} / \mathrm{m}^{2}$ years and $27.38 \mathrm{~kg} / \mathrm{m}^{2}$ respectively, with significant difference between the groups (Table 4).

\begin{tabular}{|l|l|l|l|}
\hline & One side group $(\boldsymbol{n = 6 2 )}$ & Both side group( $\boldsymbol{n = 7 3 )}$ & $\mathbf{t}(\boldsymbol{p})$ \\
\hline Age $($ year $)$ & $59.58 \pm 8.24^{\text {a }}$ & $66.95 \pm 10.20$ & $-4.638(0.000)$ \\
\hline Height $(\mathbf{c m})$ & $157.31 \pm 7.12$ & $152.34 \pm 5.68$ & $4.505(0.000)$ \\
\hline Weight $(\mathbf{k g})$ & $66.84 \pm 9.45$ & $63.62 \pm 8.97$ & $2.030(0.044)$ \\
\hline BMI $\left(\mathbf{k g} / \boldsymbol{m}^{2}\right)$ & $26.96 \pm 3.02$ & $27.38 \pm 3.38$ & $-0.760(0.448)$ \\
\hline aMean \pm SD, TKR: Total Knee Replacement, BMI: Body Mass Index & \\
\hline
\end{tabular}

\section{Table 4.}

General characteristics of participants by one side arthritis and both side arthritis $(\mathrm{N}=135)$

The ROM of knee flexion of both legs in the uniside arthritis group were 131.27 degrees (left leg) and 128.63 degrees (right leg), respectively, with significant differences between the both legs $(p<0.05)$. The range of knee flexion contracture of both legs in the Uniside arthritis group were 0.91 degrees (left leg) and 0.00 degrees (right leg), respectively, with significant differences between the both legs $(p<0.05)$. The range of genuvarus of both legs in the uniside arthritis group and the both side arthritis group were $-14.71 \mathrm{degrees}$ (left leg of uniside) and -19.44 degrees (right leg of uniside), -17.89 degrees (left leg both side arthritis) and -16.63 (right of both side arthritis), respectively with significant differences between the groups $(p<0.05)($ Table 5$)$.

\begin{tabular}{|c|c|c|c|c|}
\hline & & One side group $(n=62)$ & Both side group $(n=73)$ & $t(p)$ \\
\hline \multirow[t]{4}{*}{ Lt. leg } & Range of extension ${ }^{a}$ & $3.18 \pm 2.61^{a}$ & $4.18 \pm 4.41$ & $-1.489(0.139)$ \\
\hline & Range of flexion & $131.27 \pm 5.38$ & $128.63 \pm 8.05$ & $2.223(0.028)$ \\
\hline & Angle of flexion contracture & $091 \pm 2.90$ & $0.00 \pm 0.00$ & $2.324(0.024)$ \\
\hline & Range of varus & $-14.71 \pm 6.90$ & $-17.89 \pm 6.09$ & $2.715(0.008)$ \\
\hline \multirow[t]{4}{*}{ Rt. Leg } & Range of extension & $3.89 \pm 4.17$ & $4.51 \pm 3.89$ & $-0.446(0.657)$ \\
\hline & Range of flexion & $128.89 \pm 4.86$ & $127.25 \pm 9.92$ & $0.485(0.629)$ \\
\hline & Angle of flexion contracture & $0.00 \pm 0.00$ & $0.14 \pm 1.19$ & $-0.354(0.724)$ \\
\hline & Range of varus & $-19.44 \pm 1.67$ & $-16.63 \pm 5.90$ & $-3.145(0.003)$ \\
\hline
\end{tabular}

\section{Table 5 .}

Differences of range of motion by one side arthritis and both side arthritis $(\mathrm{N}=135)$

The VAS scores of the uniside arthritis group and the both side arthritis group were 5.92 and 6.89 , respectively, with significant differences between the groups $(p<0.05)$. The WOMAC scores of the uniside arthritis group and the both side arthritis group were 71.61 and 71.28 , respectively, with significant differences between the groups $(p<0.05)$. The SF-36 of the uniside arthritis group and the both side arthritis group were 46.19 and 43.84 , respectively, with significant differences between the groups $(p<0.05)$. The SF-36 PCS of the uniside arthritis group and the both side arthritis group were 32.22 and 30.51 , respectively, with significant differences between the groups $(p<0.05)$. The SF-36 MCS of the uniside arthritis group and the both side arthritis group were 39.29 and 38.72, respectively, with significant differences between the groups $(p<0.05)$ (Table 6).

\begin{tabular}{|l|l|l|l|l|}
\hline \multicolumn{2}{|l|}{} & TKR $(\boldsymbol{n = 7 0 )}$ & non TKR $(\boldsymbol{n = 6 5})$ & t $(\boldsymbol{p})$ \\
\hline VAS & & $5.92 \pm 1.26^{\mathrm{a}}$ & $6.89 \pm 1.21$ & $-4.550(0.000)$ \\
\hline Left leg & KSS & $46.72 \pm .75$ & $39.93 \pm 6.50$ & $4.804(0.000)$ \\
\hline & KSFS & $44.35 \pm 14.11$ & $35.68 \pm 15.86$ & $3.188(0.002)$ \\
\hline HSS & $71.04 \pm 6.78$ & $66.15 \pm 6.72$ & $4.037(0.000)$ \\
\hline
\end{tabular}




\begin{tabular}{|l|l|l|l|l|}
\hline Right leg & KSS & $37.63 \pm 4.31$ & $40.54 \pm 8.45$ & $-0.956(0.342)$ \\
\hline & KSFS & $38.13 \pm 14.87$ & $35.21 \pm 15.59$ & $0.503(0.616)$ \\
\hline HSS & $66.00 \pm 6.99$ & $66.15 \pm 7.07$ & $-0.590(0.953)$ \\
\hline SF-36 & & $76.16 \pm 6.88$ & $71.28 \pm 5.79$ & $4.479(0.000)$ \\
\hline SF-36 PCS & $46.19 \pm 4.77$ & $43.84 \pm 3.84$ & $3.181(0.002)$ \\
\hline SF-36 MCS & $32.22 \pm 3.17$ & $30.51 \pm 2.53$ & $3.472(0.001)$ \\
\hline
\end{tabular}

aMean \pm SD, KSS; Knee Society Score, KSFS; Knee Society Function Score, HSS; Hospital for Special Surgery, WOMAC; Western Ontario McMaster Universities Osteoarthritis Index, SF-36; Short Form-35 Health Survey, PCS; Physical Component Summary, MCS; Mental Component Summary

\section{Table 6 .}

The comparison of pain, function, QOL by one side and both side arthritis $(\mathrm{N}=135)$

\section{Discussion}

In this study, there were significant differences in the angles of knee extension and flexion, tibiofemoral angle and angle of genuvarus among a group with TKR which, in turn, indicates a more decrease in the range of motion among the group with TKR after the operation.

According to the reports on the range of motion of knee, the full range of motion (135 degrees) is not needed for the activities of daily living. From person to person, there could be different ranges of motion; however, it is generally known that 117 degrees are needed to tie a shoelace and to put on socks while 90 degrees are needed to sit down on a chair and about 80 degrees to climb stairs.

Nevertheless, in the case of knee extension range, it is reported that the full range of knee extension is required to carry out any activity of daily living without limitation. In this respect, it appears that the group with TKR would face limitations to carrying out the activities of daily life since the angle of their knee extension is 4.85 degrees, prohibiting full knee extension. And there were significant differences in the pain, KSS, HSS and quality of life among the group with TKR.

Knee osteoarthritis affects the structure of muscle, results in a high body mass index and induces a decrease in the range of knee flexion and quadriceps thickness while displaying the asymmetry of weight support and the limitations of gait (Strasser, Draskovits, Praschak, Quittan, \& Graf, 2013). According to Stevens-Lapsley et al. (2010), there was a significant difference in the simultaneous contraction of hamstring muscles on the knee with TKR during a maximum quadriceps contraction after comparing the muscle contraction of the knee with TKR with that of the knee without TKR (Stevens-Lapsley, Schenkman \& Dayton, 2011). Moreover, the simultaneous muscle contraction was reported to be larger in the knee with TKR than in the knee without TKR. It seems that a reduction in the muscle activity due to the muscle weakness after the TKR operation was compensated by the larger simultaneous muscle contraction while at the same time, producing low expectations of functional activities.

In fact, according to the findings of the present study, the group with TKR complained of a mid to high degree of pain with the VAS of 7.00 point which is a significant difference from that of the group without TKR. Moreover, with regard to KSS, KSFS and HSS, while the TKR group showed significant differences, the scores of their functional activities was not relatively high. The difference in the VAS, KSS and HSS among the TKR group ultimately causes a decline in the quality of life of the TKR group. As can be seen from the present study findings of WOMAC and SF-36, there is a significant difference among the TKR group, indicating a decline in the quality of life among the TKR group. This is a great issue as it violates the goal of TKR, which is to reduce pain, improve contracture and recover locomotion than before. In the comparison of the range of motion between a group with arthritis in one knee and a group with arthritis in both knees, there was a significant difference in the angles of flexion, flexion contracture and genuvarus among the group with arthritis in both knees. In addition, when comparing the range of motion of the group with arthritis in one knee with that of the group with arthritis in both knees, the group with arthritis in both knees showed great differences in the angles of flexion, flexion contracture and genuvarus.

The difference in the range of motion was similar to the differences in pain and functional state. What's more, the VAS, KSS, KSFS and HSS of the left knee indicated high pain and low functional state. The differences between the groups in the scores for pain and functional state were in line with that of the quality of life. There were significantly higher scores for WOMAC, SF-36, SF-36PCS and SF-36 MCS among the group with arthritis in both knees.

Although the scores for pain, functional state and the quality of life among the group with arthritis in one knee were better than those of the group with arthritis in both knees, there would be a high likelihood of pain and impaired functions in the unaffected knee since the affected knee could shift the weight to the unaffected knee in order to compensate the affected knee's lack of ability to support the body weight. Therefore, there appears to be a need for systematic management programs to prevent the unaffected knee from being affected by increased body weight to support.

Especially, TKR group showed a significant difference in quality of life compared to non TKR group in this study. Systematic management through the muscle strength training is needed to maintain the quality of life. The maintenance of muscle strength is the first and foremost thing which patients with total knee replacement should keep in mind and improve on in order to manage pain and knee functions and to enhance the quality of life. The rehabilitation training to recover the muscle strength of lower extremities is critical to return to the daily life before the onset of the disease (Hurtock \& Elizabeth, 1986). Since the muscle strength is one of the important physical functions, its maintenance must be factored in to rehabilitation training. After TKR surgery, muscle strength of lower extremities and co-activation of extensor and flexor of knee is important factor for early rehabilitation stage after surgery. And this factor influences the activity pattern of the muscles (Thomas et al., 2014). Also, the strong muscle group showed more stable balance ability than the weak muscle group (Butler et al., 2008). The muscle strength of lower extremities is most important factor for balance and quality life including activity daily living. In addition, there should be continuous intervention of various rehabilitation approaches to improve the angles of knee flexion on both legs since there is still a significant difference between the two legs even after a total knee replacement operation. This study has limitations in regards of participant selection. First, the numbers of participants were not enough to generalize the outcomes of the study because of the small sample size. But this study has clinical significance and implications for practical therapy due to minimized biases ranging from 
sampling and random assignment to training environment. Second, the outcomes of this study cannot be applied to all stroke patients consistently because this study focuses on chronic patients 6 months after stroke onset. Therefore, further study is needed to generalize the clinical implication of the study by varying stroke length and increasing the number of participants.

\section{References}

Butler AA, Lord SR, Rogers MW, Fitzpatrick RC (2008) Muscle weakness impairs the proprioceptive control of human standing. BrainRes 25: 244-251.

Dickstein R, Heffes Y, Shabtai El, Markowitz E (1998) Total knee arthroplasty in the elderly: patients' self-appraisal 6 and 12 months postoperatively. Gerontology 44: 204-210.

Felson DT, Anderson JJ (1987) Across-study evaluation of association between steroid dose and bolus steroids and avascular necrosis of bone. Lancet 1: 902-906.

Gidwani S, Tauro B, Whitehouse S, Newman JH (2003) Do patients need to earn total knee arthroplasty? J Arthroplasty 18 : $199-203$.

Holm B, Kristensen MT, Bencke J, Husted H, Kehlet H, et al. (2010) Loss of knee-extension strength is related to knee swelling after total knee arthroplasty. Arch Phys Med Rehabil 91: 1770-1776.

Hurtock E, Elizabeth B (1986) Development psychology, 3rd edition. McGraw Hill.

Ikejiani CE, Leighton R, Petrie DP (2000) Comparison of patellar resurfacing versus non-resurfacing in total knee arthroplasty. Can J Surg 43: $35-38$.

Keurentjes, JC, Fiocco M, So-Osman C, Onstenk R, Koopman-Van Gemert AW, et al. (2013) Patients with severe radiographic osteoarthritis have a better prognosis in physical functioning after hip and knee replacement: A cohort-study. PLoS One 8: e59500.

Ko Y, Narayanasamy S, Wee HL, Lo NN, Yeo SJ, et al. (2011) Health-related quality of life after total knee replacement or unicompartmental knee arthroplasty in an urban Asian population. Value Health 14: 322-328.

Lingard EA, Katz JN, Wright RJ, Wright EA, Sledge CB (2001) Validity and responsiveness of the knee society clinical rating system in comparison with the SF-36 and WOMAC. J Bone Joint Surg Am 83: 1856-1864.

Lundblad H, Kreicbergs A, Soderlund V, Ulfgren AK, Stiller CO, et al. (2012) The value of preoperative grade of radiographic and histological changes in predicting pain relief after total knee arthroplasty for osteoarthritis. Knee Surg Sports Traumatol Arthrosc 20: $1815-1821$.

McClelland JA, Webster KE, Feller JA, Menz HB (2010) Knee kinetics during walking at different speeds in people who have undergone total knee replacement. Gait Posture 32: 205-210.

McKay C, Prapavessis H, Doherty T (2012) The effect of a prehabilitation exercise program on quadriceps strength for patients undergoing total knee arthroplasty: A randomized controlled pilot study. PM R 4: 647-656.

Nunez M, Lozano L, Nunez E, Sastre S, Luis Del Val J, et al. (2011) Good quality of life in severely obese total knee replacement patients: a case-control study. Obes Surg 21: 1203-1218.

Russo A, Bragonzoni L, Trozzi C, Zaffagnini S, Neri MP, et al. (2008) Recurrence of varus/valgus deformity after TKR at 3 years follow-up. Knee 15: 20-25

Stevens-Lapsley JE, Schenkman ML, Dayton MR (2011) Comparison of self-reported knee injury and osteoarthritis outcome score to performance measures in patients after total knee arthroplasty. PM R 3: 541-549.

Strasser EM, Draskovits T, Praschak M, Quittan M, Graf A (2013) Association between ultrasound measurements of muscle thickness, pennation angle, echogenicity and skeletal muscle strength in the elderly. Age 35: 2377-2388.

Thomas AC, Judd DL, Davidson BS, Eckhoff DG, Stevens Lapsley JE (2014) Quadriceps/hamstrings co-activation increases early after total knee arthroplasty. Knee 21: 1115-1119.

Valtonen A, Poyhonen T, Sipila S, Heinonen A (2010) Effects of aquatic resistance training on mobility limitation and lower-limb impairments after knee replacement. Arch Phys Med Rehabil 91: 833-839. 